\title{
Norois
}

Environnement, aménagement, société

$211 \mid 2009 / 2$

Eau, pêche, tourisme rural, conflits d'usage

\section{Héritages, transformations des structures agraires et dynamique du tourisme rural en République Tchèque}

Inheritances, changes in agrarian structures and rural tourism dynamics in

Czech Republic

\section{Pascal Chevalier}

\section{OpenEdition}

Journals

Édition électronique

URL : http://journals.openedition.org/norois/2889

DOI : 10.4000/norois.2889

ISBN : 978-2-7535-1559-8

ISSN : 1760-8546

Éditeur

Presses universitaires de Rennes

Édition imprimée

Date de publication : 1 octobre 2009

Pagination : 53-65

ISBN : 978-2-7535-0937-5

ISSN : 0029-182X

Référence électronique

Pascal Chevalier, « Héritages, transformations des structures agraires et dynamique du tourisme rural en République Tchèque », Norois [En ligne], 211 | 2009/2, mis en ligne le 01 octobre 2011, consulté le 19 avril 2019. URL : http://journals.openedition.org/norois/2889; DOI : 10.4000/norois.2889

(c) Tous droits réservés 


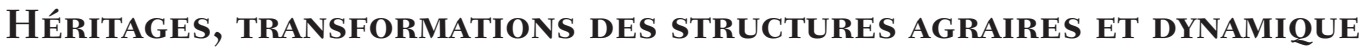 du tourisme ruRal en RÉpublique Tchèque
}

\author{
Pascal Chevalier \\ FRE 3027 - CNRS - ART-Dev \\ (Université Montpellier III), \\ Département de géographie et aménagement, Route de Mende - 34199 Montpellier Cedex 5, France \\ pascal.chevalier@univ-montp3.fr
}

\section{RÉSUMÉ}

Le développement des activités touristiques et de loisirs est un des éléments les plus perceptibles de la diversification socio-économique des espaces ruraux tchèques. Depuis le début des années 1990, le tourisme rural tchèque s'est fortement adossé à l'activité agricole qui lui sert encore aujourd'hui de pilier et de levier. Par cette forte liaison, il a été, comme il l'est encore aujourd'hui, très dépendant des changements qui se sont opérés dans le monde agricole. Les héritages de l'époque communiste, la décollectivisation et ses modes d'application sur les différents types de structures agraires, l'adaptation à l'économie de marché et l'intégration à l'Union européenne, ont certes transformé les structures d'exploitation et la société rurale en général, mais ont également contribué à l'avènement de différentes formes de tourisme rural. Cet article se propose d'analyser l'impact des héritages et des transformations agraires dans la nouvelle structuration du tourisme tchèque. À l'échelle nationale, il est question d'analyser les éventuelles corrélations existantes entre les dynamiques agricoles et touristiques et de comprendre l'impact des unes sur les autres.

MotS CLÉ : République Tchèque - tourisme rural - agriculture - structures agraires - décollectivisation - diversification

\section{ABSTRACT \\ Inheritances, changes in agrarian structures and rural tourism dynamics in Czech Republic}

The development of tourist and leisure activities is one of the most perceptible elements of the socioeconomic diversification of the Czech rural areas. Since the beginning of the 90 s, the Czech rural tourism strongly leaned on the agricultural activity. This one is a mainstay and leverage for agricultural development. By this strong connection, it was, and even is today, very dependent on changes which took place in the agricultural world. Inheritances of communist period, decollectivisation and its methods of application on the various types of agrarian structures, adaptation to the market economy and integration to the European Union, certainly transformed the agritourism enterprises structures and the rural society, but also contributed to emergence of various forms of rural tourism. This article suggests analyzing the impact of the inheritances and the agrarian transformations in the new structuralization of the Czech tourism. On a national scale, it is question to analyze the possible existing correlations between the agricultural and tourist dynamics and to understand the impact of the some on the others. 


\author{
KEY WORDS : Czech Republic - rural tourism - agriculture - agrarian structure - col- \\ lectivism - diversification
}

Depuis le début des années 1990, la problématique des trajectoires d'évolution des campagnes est au centre de l'analyse des transformations du tissu économique en République Tchèque. La rupture politique majeure de 1989 et l'intégration du pays à l'Union Européenne en 2004 ont conduit la société rurale tchèque à formuler une grande variété de réponses aux exigences imposées par les nouvelles demandes du marché (Zrinscak, 2000). Les profondes mutations sociales et culturelles, en partie légitimées et accompagnées par les projets politiques nationaux et européens, ont modifié tant l'usage des campagnes tchèques, affectées selon les régions toujours davantage à la résidence, au loisir et au tourisme, que les regards de la société portés sur les espaces ruraux euxmêmes. Ces nouveaux usages des campagnes ont transformé, avec plus ou moins d'importance, les systèmes socioéconomiques locaux en orientant une partie de l'emploi rural, jusqu'alors dominé par l'agriculture, vers des activités de tourisme et d'accueil. Bien évidemment, la fréquentation touristique des campagnes tchèques n'est pas un fait nouveau, mais l'intégration socioéconomique des espaces ruraux par le développement touristique se lit désormais dans l'augmentation de la capacité d'hébergement. Elle va de pair avec l'amélioration des infrastructures de communication et la diversification de l'offre de produits touristiques. Elle s'appuie en particulier, dans le secteur marchand, sur une économie de services qu'elle contribue dans le même temps à dynamiser.

Aujourd'hui, à l'échelle du pays, cette dynamique des activités touristiques et de loisir dessine de forts contrastes spatiaux. Certes, si le différentiel des potentialités paysagères et les héritages culturels régionaux sont souvent à l'origine de la situation, ils ne sauraient, à eux seuls, constituer l'unique réponse à des questionnements qui doivent prendre en compte d'autres facteurs territoriaux (proximité de la clientèle, accessibilité aux marchés, spécificités des structures sociales, culturelles et économiques des territoires, degré d'implication des acteurs, etc.). Parmi ces derniers, ceux liés à l'agriculture et à sa transformation sont très importants dans un pays où, le tourisme, perçu comme une alternative à la crise des exploitations agricoles après le changement de système politique au début des années 1990, s'est fortement adossé à l'activité agricole qui lui sert aujourd'hui de pilier et de levier. Par cette forte liaison, il a été, comme il l'est encore aujourd'hui, très dépendant des changements qui se sont opérés dans ce secteur économique. Cette étude ${ }^{1}$ vise à analyser l'impact de ces transformations dans la nouvelle structuration du tourisme tchèque. Dans un premier temps, il est question de faire un état des lieux de l'activité touristique tchèque et d'en comprendre les principaux facteurs de structuration. Ensuite, dans un second temps, il s'agit, à partir des différentes dynamiques agricoles et touristiques, d'analyser d'éventuels liens entre les deux activités et d'en comprendre l'articulation actuelle.

\title{
Fonction touristique et contrastes spatiaux
}

En République Tchèque, comme dans la plupart des pays européens, les loisirs sont devenus, avec la santé, un des postes de dépense ayant le plus progressé. En volume, les dépenses de loisirs ont été multipliées par 6 entre 1989 et $2006^{2}$ alors, qu’à titre d'exemple, elle n'a été multipliée que par 1,5 dans la plupart des pays occidentaux. Parallèlement, grâce à une clientèle tchèque au pouvoir d'achat nettement réévalué depuis la chute du communisme et à une clientèle étrangère plus fortunée, composée essentiellement d'Allemands et d'Autrichiens venant chercher des services aux coûts inférieurs à ceux pratiqués dans leurs pays d'origine, l'évolution annuelle de la consommation en loisirs s'est largement développée. Portée par les goûts actuels plus proches de

1. Cet article est issu d'un travail de recherche intitulé «Action locale et dévellopement territorial en Europe centrale », $n^{\circ}$ ANR-08-BLAN-0270-01 financé par l'Agence nationale de la recherche.

2. Office-Cesky Statisticky Urad (CSU), fichier emploi, 2006. 

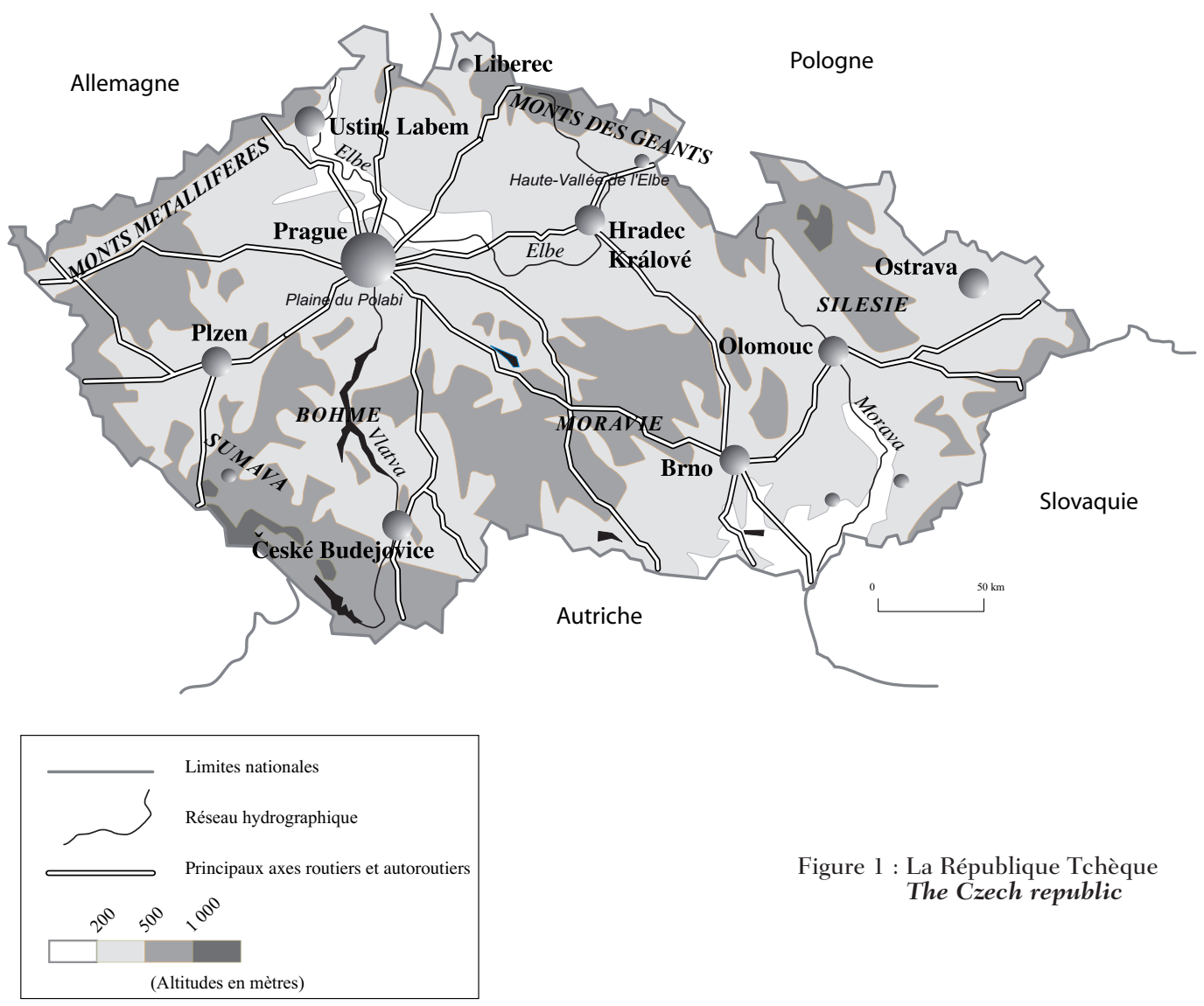

Figure 1 : La République Tchèque The Czech republic

l'environnement, elle s'est transformée et a permis à l'espace rural de se positionner favorablement sur un marché du tourisme en plein essor. Un grand nombre d'éléments l'atteste comme l'augmentation, certes moyennement significative, des structures d'accueil traditionnelles (hôtellerie classique), mais surtout l'augmentation assez importante des exploitations concernées par l'agritourisme ou d'autres formes d'accueil rural ${ }^{3}$. Ceci dit, l'attractivité des régions rurales tchèques n'est pas homogène et si ces dynamiques tendent à s'étendre spatialement, elles ne s'inscrivent pas uniformément dans les territoires.

\section{UNE INTENSITE TOURISTIQUE VARIABLE SELON LES REGIONS}

L'intensité touristique est un indicateur très utile pour connaître l'ouverture des campagnes vers ce secteur d'activité économique. Elle correspond au nombre de nuitées touristiques pour 10000 habitants dans l'unité administrative considérée. Ceci dit, il faut s'entendre d'abord sur le type de nuitées recensées : certaines statistiques incluent les hébergements légers (gîtes, refuges, campings, auberges de jeunesse), d'autres non. Mais ensuite, bien des flux échappent à cette comptabilité officielle : en particulier, la République tchèque est marquée par l'existence de nombreuses résidences familiales secondaires en milieu rural, les Chalupas. Sous le socia- 
lisme déjà, il s'agissait de petits bâtiments rustiques en auto-construction, entourés d'un jardinet d'agrément, équivalent des datchas russes. L'intensité touristique ne traduit qu'un enregistrement des flux officiels, nationaux et internationaux, ce qui accentue artificiellement les contrastes entre régions. En effet, si l'intensité touristique apparaît extrême dans certaines régions occidentales du pays et très faible dans d'autres, c'est essentiellement parce que le tourisme officiel, seul recensé, est plus concentré que le tourisme léger et familial de certains secteurs ruraux ${ }^{4}$.

Les activités touristiques se structurent autour de quelques pôles de forte fréquentation, mais continuent d'ignorer de vastes espaces. Elles font apparaître des contrastes spatiaux très marqués qui soulignent la marginalisation de vastes régions rurales de la partie orientale et industrielle du pays. Dans les régions de Novy Jicin et d'Ostrava où la présence d'anciennes activités minières et manufacturières, aujourd'hui en crise profonde, se lit encore très nettement dans les paysages, le nombre de nuitées pour 10000 habitants dépasse à peine les 900 par an.

Ailleurs, tandis que les phénomènes de diffusion restent limités au-delà des zones traditionnelles de séjour, des pôles urbains et de quelques sites remarquables (lac du Paradis bohémien, etc.), l'activité s'amplifie au fur et à mesure que l'on se rapproche des frontières occidentales du pays. Les régions à proximité de Prague, véritable moteur de l'activité touristique tchèque, bénéficient d'une attractivité ancienne et soutenue. De la région de Beroun jusqu’à celle de Nymburk, en passant par Praha-zapad et Praha-Vychod, les scores de fréquentation sont élevés et dépassent parfois, dans les communes les mieux reliées à la capitale tchèque, plus de 20000 nuitées/10000 hab. par an. Il en est de même dans les pôles touristiques traditionnels de Moravie où, avec environ 30000 nuitées/ 10000 hab/an, ils ont des effets comparables sur le tissu socioéconomique local.

Au nord-est du bassin agricole du Polabí, dans la région de Liberec et malgré la modestie des altitudes des massifs hercyniens, la continentalité du climat a favorisé, dès l'époque socialiste, l'implantation de stations de sports d'hiver dont la fréquentation ne semble pas moins importante que dans celles des massifs plus enneigés d'Europe centrale (Carpates polonaises et Grandes Tatras slovaques). Certes, si le changement de système a profondément déstabilisé les structures les moins adaptées aux exigences croissantes de la clientèle, les importants investissements consentis par les institutions européennes et tchèques (ministère du Tourisme et de l'Aménagement), les pouvoirs locaux (régions, municipalités) et des grands groupes industriels allemands, ont profondément transformé les établissements en les modernisant et en les adaptant aux nouvelles normes de confort attendues par des clientèles essentiellement autrichiennes et allemandes.

\section{UNE OFFRE TOURISTIQUE QUI SE DIVERSIFIE}

Plus anciens que les sports d'hiver, le thermalisme et le climatisme, dont les pratiques remontent pour l'essentiel au milieu du XIX ${ }^{\mathrm{e}}$ siècle, continuent à attirer des clientèles dans le nord-oust du pays (région de Hradec-Kralové) et dans la partie méridionale des Hautes-terres tchéco-moraves. Déjà, en 1920, dans la Haute-Vallée de l'Elbe, on comptabilisait plus de 80000 curistes par an et cet engouement, même pendant l'époque communiste où les dirigeants du parti venaient en famille se détendre dans les bains de Kuks ou de Dvur Kralové, ne s'est jamais tari. Depuis le changement de système - et en partie grâce aux Fonds Structurels Européens de pré adhésion $\left(\mathrm{SAPARD}^{5}\right)$ et au Programmes d'Initiative Communautaire LEADER ${ }^{6}-$ la réalisation d'importants programmes d'aménagement et de modernisation des stations thermales a profondément

4. Pour compenser ce déficit d'enregistrement, nous avons donc eu recours dans cette première cartographie de l'intensité touristique à une transformation des intensités « officielles » par le logarithme décimal, de telle sorte à en atténuer quelque peu les contrastes et permettre la prise en compte de certains sites au tourisme certes bien moins intense, mais néanmoins significatif par rapport à la moyenne nationale. Là encore, les contextes régionaux sont donc très différents entre eux et affectés par le mode de collecte de l'information statistique.

5. Le programme SAPARD de préadhésion a pour objectif, notamment, de résoudre les problèmes prioritaires liés à l'adaptation à long terme des économies des pays candidats et de faciliter la mise en ouvre par ceux-ci de l'acquis communautaire, en mettant l'accent sur la politique agricole commune.

6. Liaisons Entre Actions de Développement de l'Économie Rurale. 

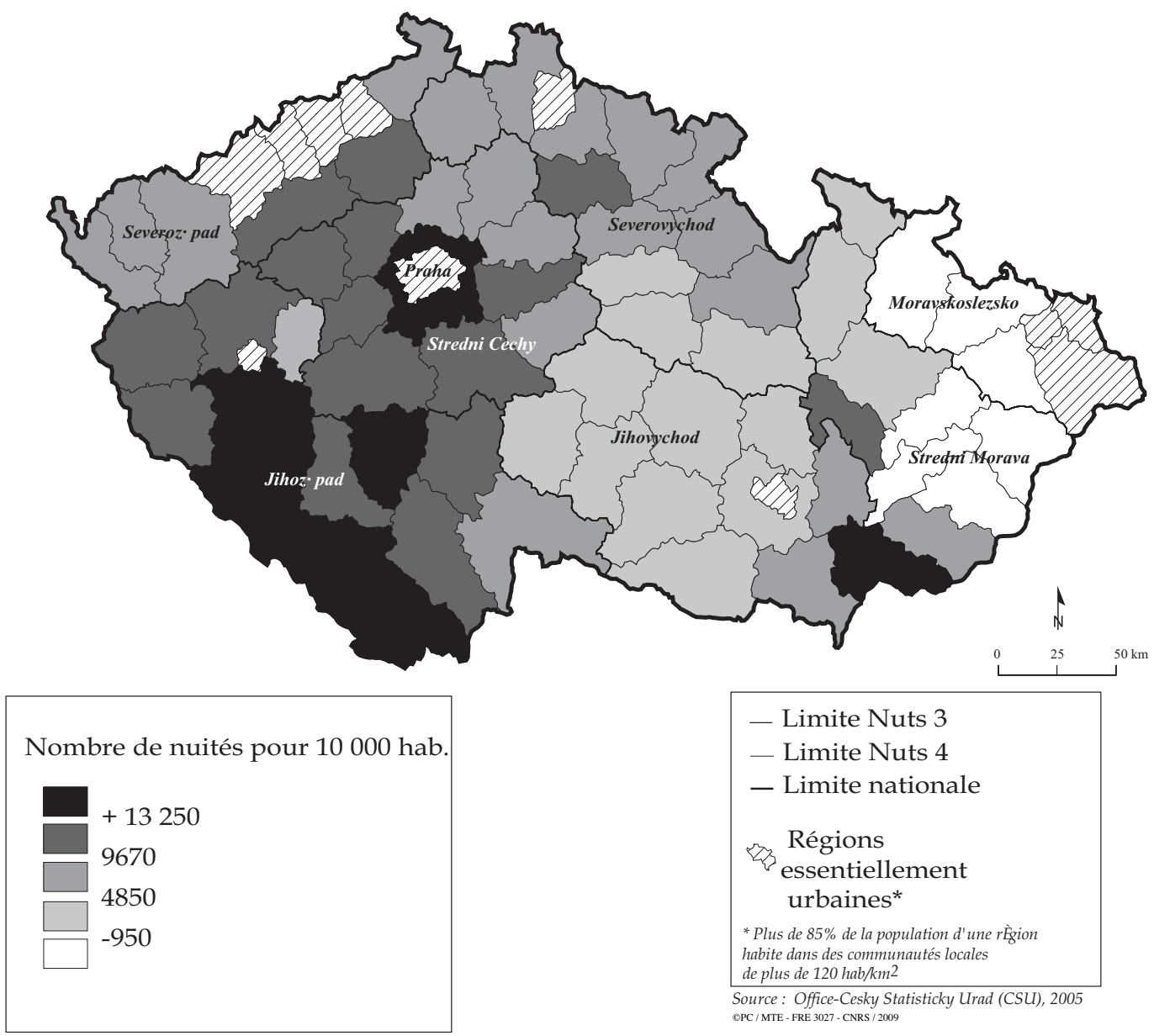

_ Limite Nuts 3

— Limite Nuts 4

- Limite nationale

27 Régions

essentiellement urbaines*

* Plus de $85 \%$ de la population d'une rÈgion habite dans des communautés locales

de plus de $120 \mathrm{hab} / \mathrm{km} 2$

Source: Office-Cesky Statisticky Urad (CSU), 2005 OPC / MTE - FRE 3027 - CNRS / 2009

Figure 2 : Densité touristique en République Tchèque en 2005

Tourist intensity in Czech republic in 2005

modifié la physionomie d'une activité qui s'est largement diversifiée ${ }^{7}$. À Marienbad et à Karlsbad en Bohème septentrionale, le long de laquelle s'égrènent les stations les plus anciennes du pays et les plus fréquentées pendant la période du régime socialiste, la demande étrangère et l'injection de capitaux occidentaux ont permis d'intensifier les activités thermales d'agréments. Dans les Monts des Géants, à la frontière polonaise, c'est un thermalisme sanitaire, soutenu par les politiques publiques nationales et spécialisé dans le traitement des affections articulaires qui s'est fortement développé. En Moravie méridionale, les exigences imposées par le marché ont contraint les acteurs locaux (élus, associations et entrepreneurs) à proposer, dans leurs établissements, des services mixtes (agrément et sanitaire) pour bénéficier de doubles marchés et élargir leurs clientèles. Enfin, dans le sud du pays et dans les Carpates Blanches, où les stations thermales les plus vétustes du pays ont connu un déclin soutenue de leur activité, la présence d'un important patrimoine immobilier et d'une main-d'œuvre spécialisée ont permis, grâce à l'aide de l'Etat, de

7. Outre le programme LEADER financé par l'Union Européenne, il existe un programme LEADER tchèque, financé par l’Etat, et géré directement par le Ministère de l'Agriculture. 
réorienter partiellement les services vers de nouveaux secteurs sanitaires et sociaux (centres pour handicapés, centres gériatriques spécialisés).

Le tourisme vert et de pleine nature constitue un volet déterminant de l'activité touristique tchèque (Majerová, 2000). Comme dans une très grande partie des pays européens, le changement des modes de consommation des clientèles a offert de nouvelles opportunités dans le développement de formes de tourisme plus proche de l'environnement. Les prestations proposées sont diverses avec une nette prépondérance pour « la formule gîte ». Là aussi, selon un net gradient ouest/est, l'offre en gîtes, chambres d'hôtes et autres fermes auberges, correspond en grande partie aux territoires occidentaux tchèques (Šumava) et de quelques terroirs épars en Moravie ou dans les Sudètes, désormais attractifs pour l'esthétique de leurs paysages agraires, le pittoresque de certains de leurs sites naturels et pour la pratique des activités de plein air (randonnées pédestres, cyclistes, équestres, sports en eau vive, etc.). Les fermes pédagogiques rencontrent également un succès non négligeable puisque près d'un exploitant agritouristique sur cinq ${ }^{8}$ s'est lancé, ces dernières années, dans ce type de service. Ces exploitations sont situées essentiellement dans les provinces proches de Prague ou de Brno.

\section{Dynamiques touristiques et dynamiques agraires : quels liens?}

Depuis le début des années 1990, le tourisme rural s'est fortement adossé à l'activité agricole, soit directement, en utilisant les structures des exploitations par le biais de l'agritourisme ${ }^{9}$, soit indirectement en appuyant et valorisant les productions locales. La place de cet agritourisme dans le tourisme rural est d'ailleurs très significative. Globalement, en 2005, la part de l'hébergement agritouristique dans le nombre total de lits touristiques au niveau national est supérieure à $40 \%$. Dans certaines régions, les plus occidentales, ce taux peut largement dépasser les $70 \%$, voire les $80 \%$ dans la région de Klatovy près de la frontière allemande. Par ces fortes liaisons, le tourisme rural a été, comme il l'est encore aujourd'hui, très dépendant des changements qui se sont opérés dans un monde agricole en pleine mutation. Ce dernier, rompant avec le système collectiviste qui avait encadré son développement pendant près d'un demi-siècle, a accompli une décollectivisation dont les effets sur les structures, les capacités de production et la diversification des activités rurales ont été brutaux et parfois destructeurs (Majerová, 2000).

Dans cette deuxième partie, il est question de percevoir les éventuelles liaisons entre les dynamiques agricoles et touristiques et d'en comprendre les articulations. Une analyse multi variée conduite à partir des différents indices touristiques et agricoles ${ }^{10}$ permet un découpage du territoire tchèque en fonction des liens (ou non liens) qui existent entre les deux fonctions agricoles et touristiques.

8. Office-Cesky Statisticky Urad (CSU), recensement agricole, 2006. Sont considérées comme fermes pédagogiques dans la nomenclature tchèque, les exploitations agricoles ayant passé une convention avec des établissements scolaires ou sociaux.

9. Dans la nomenclature tchèque, les prestations agritouristiques sont des activités de tourisme et de loisirs proposées par un ou plusieurs agriculteurs ou membre(s) de l'exploitation dont le siège est une exploitation agricole en activité (au sens juridique du terme). Ces prestations sont multiples et concernent la location d'hébergements (chambres d'hôtes ou gites) et une gamme de prestations incluant des activités d'animation (fermes pédagogique) et de restauration.

10. L'ensemble des analyses spatiales aboutissant à cette typologie associe les critères suivants :

- Nombre d'actifs par entreprise agricole et touristique (en 2005) et évolution rapportés à 100 habitants entre 1995 et 2005.

- Type de structures touristiques (hôtellerie traditionnelle, structures agritouristiques) et agricoles (taille en ha) et évolution entre 1995 et 2005 .

- Productivité des exploitations et orientation de leur production.

- Revenus agricoles et revenus touristiques

- Nombre de travailleurs indépendants, chefs d'entreprises, salariés et caractère stable ou partiel des emplois exercés. 


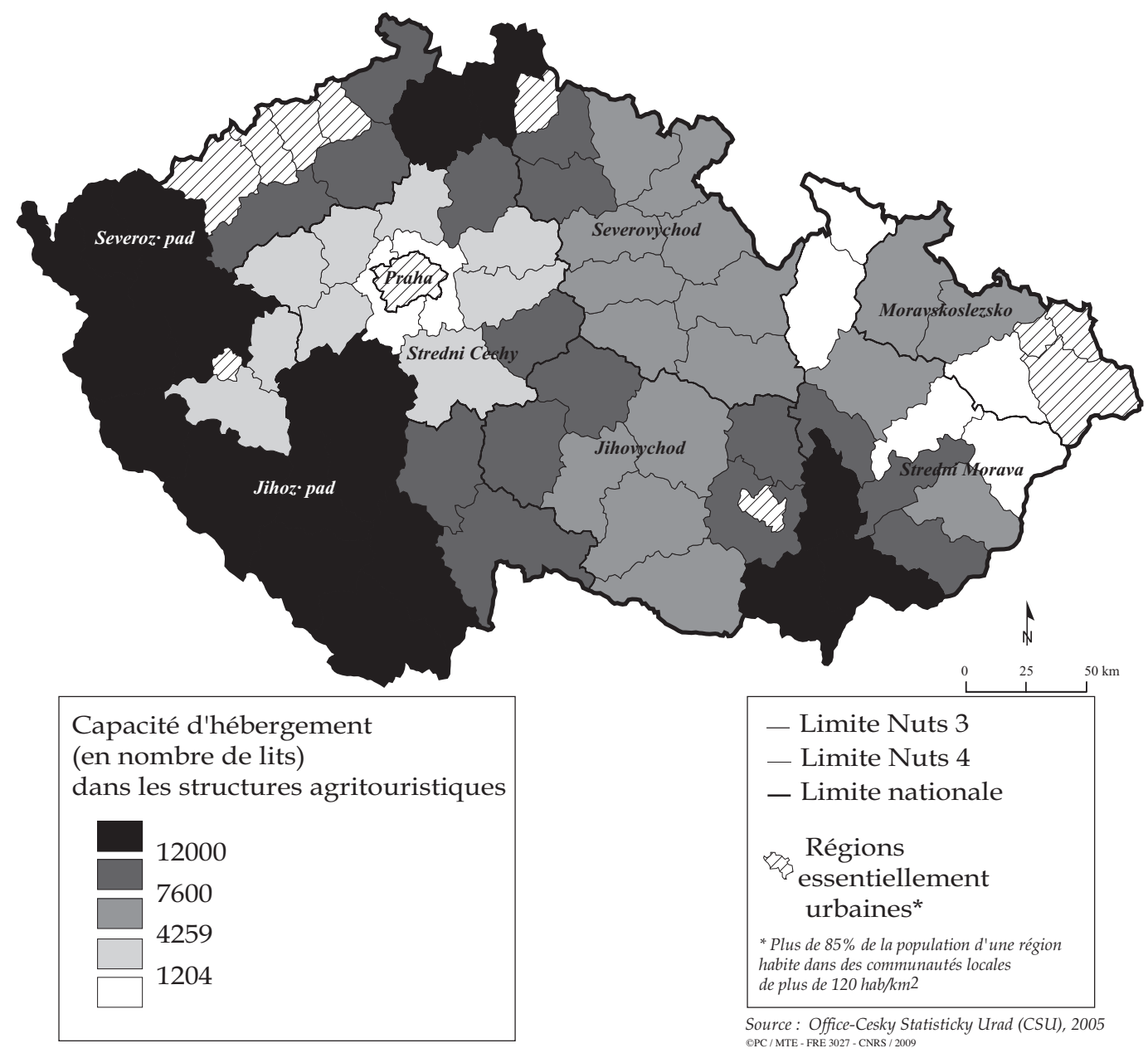

Figure 3: Capacité d'hébergement des structures agritouristiques en République tchèque en 2005 Tourist accommodation capacity in agritourism enterprises in Czech republic in 2005

\section{EN BOHÊME CENTRALE, UNE DYNAMIQUE TOURISTIQUE INDÉPENDANTE DE L'AGRICULTURE}

Dans le type 1, la corrélation entre la dynamique touristique (en volume de fréquentation) et agricole (en valeur de la production) est l'une des plus faibles du pays (0,29). L'activité touristique, largement indépendante des structures d'exploitation, est surtout orientée vers le loisir dans des établissements d'accueil relativement classiques (hôtels, auberges) et dans des centres de tourisme social (auberges de jeunesse). Dans ces terres très fertiles du bassin du Polabí, l'agriculture, aujourd'hui modernisée, n'a jamais constitué le support d'une quelconque activité d'accueil. Fortement rémunératrice, elle n’a que très peu cherché dans le tourisme des moyens de compenser un niveau revenu de la production qui a plus que quadruplé en dix ans. 


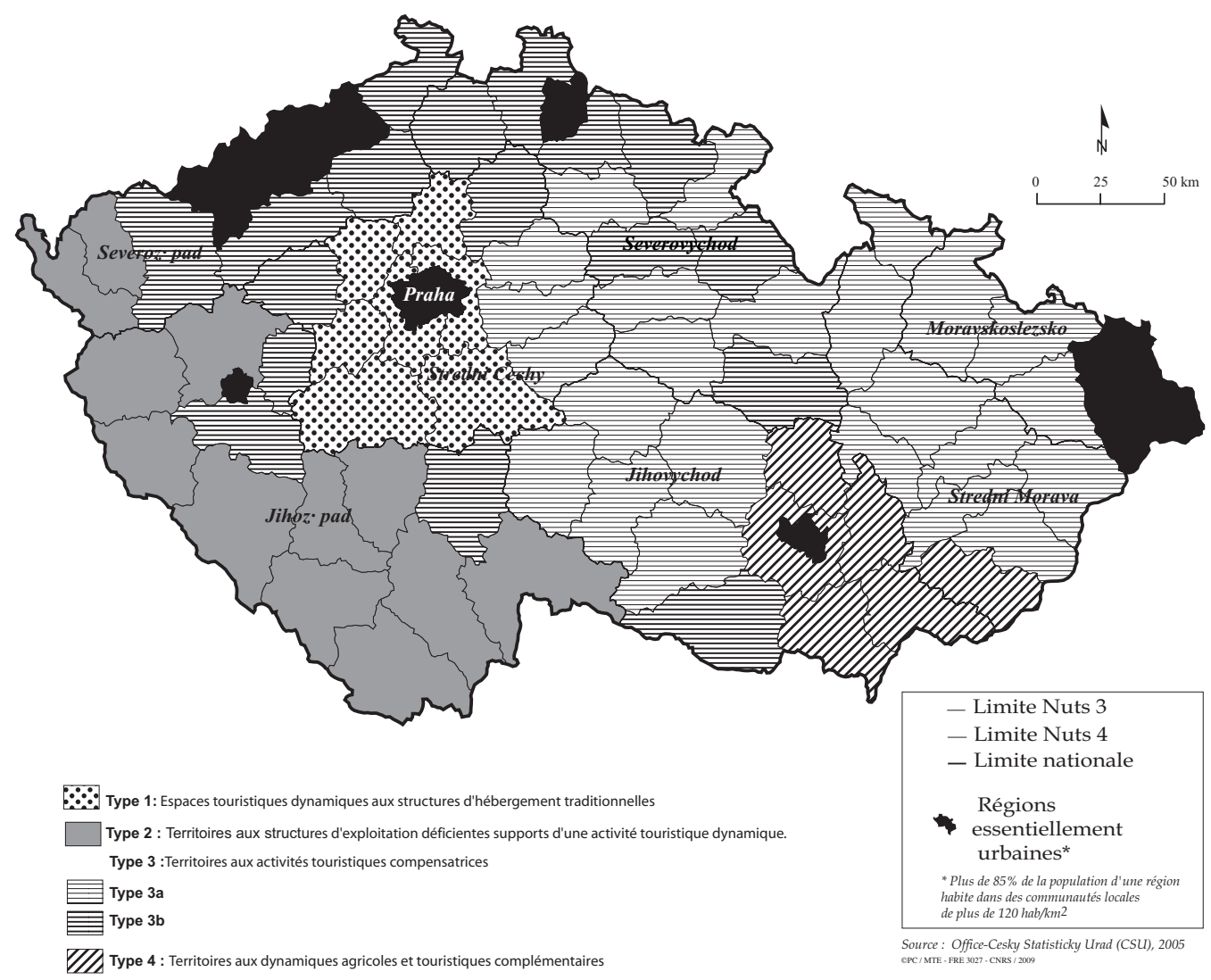

Figure 4 : Liens agriculture/tourisme rural Agriculture/rural tourism links

\section{EN Sumava, un tourisme rural qui SupPlante unE aCtivité agricole défaillante}

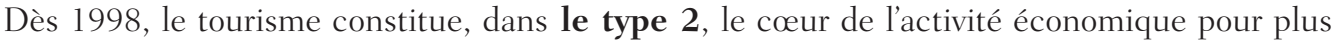
de $78 \%$ des exploitations agricoles. Lintroduction des prestations touristiques en leur sein ne s'inscrit pas spécialement dans l'optique d'une diversification agricole pour compenser des revenus en baisse, mais dans l'opportunité qui se présente de faire du tourisme. En effet, les exploitations agricoles fonctionnent majoritairement selon une logique commerciale orientée vers la réalisation de profits économiques tirés quasi exclusivement de l'activité touristique. L'activité agricole, très marginale et profondément déstructurée par les politiques de privatisation des fermes d'État, a été largement supplantée par le tourisme qui, s’il bénéficie d'avantages territoriaux indéniables (paysages de montagne, richesse de patrimoine architectural et culturel local), est favorablement localisé à proximité de la Bavière. La faible rente naturelle ayant orienté les exploitations vers des systèmes d'élevages extensifs, l'activité agricole sert assez souvent uniquement de statut social ou de vitrine pour l'exploitation touristique. Avec un coefficient de corrélation proche de -0,80 entre l'évolution de la valeur de la production agricole et celle du tourisme (en volume), la productivité agricole est inversement proportionnelle à celle du tourisme. Si $89 \%$ des unités de production intensive (qui ne représentent que $9 \%$ des exploitations), ne sont pas concernées par l'agritourisme, $92 \%$ des plus extensives développent intensément des services touristiques annexes. 
Dans ces espaces périphériques, vidés des minorités allemandes au lendemain de la Deuxième Guerre mondiale et repeuplés par des colons tchèques, le secteur d'Etat occupait une place particulière dans la mise en valeur de zones défavorisées du point de vue des conditions naturelles et détenait un rôle de contrôle de la frontière. En 1989, la privatisation des fermes d'Etat a procédé au démantèlement des exploitations géantes au profit de sociétés anonymes prenant en gérance les biens fonciers et non fonciers, dans une région où, par ailleurs les demandes de restitution de terres à exploiter sont restées assez faibles (les anciens propriétaires d'origine allemande, ayant été expulsés). Les conditions environnementales, assez contraignantes n'incitaient pas à l'installation d'exploitations individuelles. Assez vite, beaucoup de nouvelles structures sociétaires (essentiellement tournées vers l'élevage laitier) se sont heurtées aux réalités économiques. Dès 1995, les faibles rendements agricoles, les difficultés financières et les possibilités limitées de reconversion économique, ont poussé bon nombre de structures à la faillite et à la revente de leur outil de production à de petits particuliers, plus intéressés par le potentiel du patrimoine architectural et immobilier (épargné par le régime socialiste) et sa localisation à proximité de la Bavière touristique que par la production agricole. Aujourd'hui, ce patrimoine réhabilité et transformé, en partie grâce au programme européen LEADER $+{ }^{11}$ propose une combinaison de plusieurs prestations touristiques, telles que du logement, des visites de l'exploitation agricole (fermes pédagogiques) et de la restauration.

\section{Sur les Haute-Terres tCheco-moraves, un tourisme rural de COMPENSATion}

Même si l'activité touristique est présente dans certaines unités et peut être déterminante dans le maintien de l'exploitation, la production agricole reste, dans le type 3, la raison d'être de l'exploitation et sa source principale de revenus. Ici, le but recherché par les agriculteurs et celui de dégager des revenus complémentaires à l'exploitation agricole, soit parce ces derniers ont baissé, soit parce que les besoins des ménages se sont accrus. Globalement, les exploitants agricoles sont avant tout motivés par la nécessité d'un nouveau revenu qui leur permette de maintenir une exploitation agricole en partie défaillante. Septiques par rapport au futur de leur agriculture dans le contexte d'évolution de la politique agricole commune, dont l'adaptation sur le modèle intensif après 1989 s'est faite très difficilement (faible délestage de la main-d'œuvre agricole, faible valeur ajoutée de la production), déstabilisés par les rendements fluctuants, et en l'absence de capitaux suffisants pour investir dans des unités de productions plus rentables, ils dénotent une volonté d'autonomie vis-à-vis des logiques économiques globales qui leur échappent. Deux sous-classes se démarquent.

- Le type 3a: Si dans certains bassins agricoles les plus performants, la modernisation de la production agricole sur le modèle intensif dominant a pu s'effectuer au prix d'un important délestage de la main-d'œuvre et d'une relocalisation sélective des unités de production, la faiblesse de la rente naturelle et des activités de substitution dans l'industrie et les services n'ont que partiellement réglé le problème du sur-emploi agricole et maintenu un grand nombre de petites exploitations dont la plupart se sont repliées sur une économie quasi autarcique. Ces exploitations de petite taille (en moyenne 8 ha) ${ }^{12}$ et dégageant des revenus relativement bas (moins de 4000 euros en moyenne par an), mais encore très nombreuses, ont été souvent contraintes de s'orienter vers la

11. L'initiative communautaire LEADER I, introduite en 1991-1993 par la Communauté économique européenne qui comptait alors douze membres, a été reconduite une première fois dans le cadre du programme LEADER II, pour cinq ans (1995-1999), sous la forme d'interventions pilotes destinées à stimuler les approches innovantes au niveau local, puis renouvelée à partir de 2000, sous la dénomination LEADER+. Au fur et à mesure des élargissements successifs, l'expérience a été étendue à de nouveaux Etats membres. Les travaux consacrés à l'initiative communautaire LEADER mettent l'accent sur son caractère innovant et y voient une nouvelle étape du développement des zones rurales en Europe. L'originalité de LEADER concerne avant tout la méthode sur laquelle reposent la prise de décision et les actions qui en découlent. Dans une démarche de caractère ascendant, la méthode LEADER se fonde sur les principes de subsidiarité et de partenariat. Les décisions concernant la stratégie de développement local ainsi que les projets sont prises par des organes situés au plus près des périmètres d'intervention.

12. La taille moyenne des exploitations en République tchèque est de 26 hectares en 2005, mais plus de la moitié ont des superficies inférieures à une dizaine d'hectares. 
pratique d'un agritourisme faiblement compensateur, plus ou moins organisé et professionnalisé, et encore très peu encadré. Ici, grâce à une source de revenus complémentaires tirés du service touristique, la diversification est une stratégie de développement qui consiste à varier et élargir la gamme des produits exploités et des clients sollicités pour se protéger, avant toute chose, des conjonctures de l'agriculture. Tantôt ayant un objectif d'autoconsommation familiale, tantôt orientées vers les revenus supplétifs du tourisme, ces structures ont plutôt tendance à perpétuer le fonctionnement du lopin individuel du système socialiste et d'un agritourisme de très petite taille (Kučerová, 2004) dont la dynamique reste à ce jour très limitée.

- Le type 3b : Comme en Šumava, ces régions frontalières septentrionales ont connu la même série d'évènements, les expulsions allemandes, une collectivisation forcée et l'instauration des fermes d'Etat qui ont marginalisé durablement les exploitants indépendants et ont multiplié les exploitations à temps partiel. Dans ces dernières, sans révéler de véritables projets professionnels choisis et cohérents, l'agritourisme de compensation, souvent en inadéquation avec les réalités des marchés locaux, semble constituer, à l'inverse du type 3a, les bases d'une activité qui, au fil du temps, s'est structurée et s'est diversifiée. Dans des régions bénéficiant d’importantes aménités paysagères et d'une situation frontalière plus favorable que celle du type précédent, les taux de fréquentation se sont nettement élevés depuis 2000. Si la dynamique touristique engagée est encore relativement faible, les activités d'accueil commencent à être comprises comme des stratégies d'innovation menées par des exploitants agritouristiques ne concevant plus l'agriculture comme une activité totalement rentable à elle seule. Parallèlement, avec l'émergence de nouvelles productions agricoles extensives localisées, essentiellement autour de l'élevage bovin et ovin, cette forme de tourisme participe à une « ré activation » d'un lien produit/territoire (par le développement de stratégies qualitatives et de promotion des productions de terroir) fortement bousculé, pendant l'ère communiste, par la présence des fermes d'Etat mais rendu possible par l'effondrement, dans le système économique libéral, du modèle agricole intensif après 1995.

\section{UNE DYNAMIQUE TOURISTIQUE ET AGRICOLE FORTEMENT CORRÉLÉE EN MORAVIE DU SUD}

Le type 4 est caractérisé par une corrélation positive significative $(+0,67)$ entre une dynamique agricole (en valeur de la production) relativement favorable en terme social et économique (faible déclin de la population active agricole et maintien de la productivité) et le développement d'un tourisme rural (en volume de fréquentation) fortement axé sur l'agritourisme. Dans cette région, face aux rouages de la standardisation agricole sur le modèle intensif, bien représenté dans les terres les plus fertiles de la partie centrale du sillon de la Morava mais ignorant une vaste partie du territoire régional (piedmonts, zones centrales de collines), des formes plus originales de recomposition productive se sont mises en place après 1989. Héritées du maintien des rares exploitations privées de très petites tailles qui avaient échappé à la collectivisation (et qui constituaient déjà, bien avant 1989, une alternative au modèle agricole coopératif et intensif), elles sont à l'origine d'encrages territoriaux souvent fondamentaux pour l'activité touristique locale (Doucha et al., 2005).

Dans le contexte de mondialisation des économies, aussi bien dans le domaine de la production que dans celui de la consommation, on observe dans ce type 4 une remontée en puissance et spectaculaire des productions agricoles extensives localisées. Certes, en dépit de leur faible intégration dans les marchés nationaux ou internationaux en raison de leurs coûts de production encore assez élevés, elles s'inscrivent désormais dans une dynamique touristique locale dont elles constituent le soubassement et en retire des bénéfices économiques réels. Dans ce cadre, la recherche de la spécificité locale met l'accent sur un codéveloppement agricole et touristique prenant appui sur la petite exploitation familiale, la mobilisation des ressources locales, aussi bien humaines que matérielles, l'élaboration d'avantages concurrentiels liés au mouvement de re-territorialisation et de valorisation des externalités territoriales. Cela se traduit par une modification de la notion de label, avec une territorialisation plus large et plus efficace des productions locales. Ces nouveaux 
liens avec le territoire peuvent prendre de multiples aspects allant de la simple référence à l'espace support de la production pour le produit élaboré, jusqu'à une association et même l'intégration de l'espace de production au bien ou au service. On peut citer en exemple la route des vins en Moravie du Sud qui a permis, d'une part, de dynamiser et professionnaliser l'offre d'hébergement régional et, d'autre part, de relancer la production vitivinicole par le rétablissement de la vente directe et des filières de commercialisation courtes auprès de la clientèle touristique locale. Dans toutes ces démarches, les principales composantes des tendances touristiques sont à mettre en lien avec le concept d'authenticité que les touristes associent à la culture morave locale, à la rencontre avec les populations locales, aux produits du terroir, à l'immersion dans la nature, etc. Dans cette perspective, même si l'espace rural ne se confond plus avec le monde agricole, ce dernier occupe, dans le jeu des représentations, une place non négligeable au sein du dispositif patrimonial et touristique. Ces observations sont autant d'éléments qui semblent encourageants pour les agriculteurs désireux de se diversifier par l'investissement dans le tourisme. Ce contexte théoriquement favorable à la diversification agricole par le tourisme engendre de nouvelles demandes à l'égard des agriculteurs, dont la place dans la société se trouve changée.

\section{Conclusion}

La fréquentation touristique des campagnes n'est pas un fait nouveau. L'intégration socio-économique des espaces ruraux par le développement touristique se lit désormais dans l'augmentation de la capacité d'hébergement, la rénovation des infrastructures hôtelières et la multiplication des chambres d'hôtes. Allant de pair avec l'amélioration des infrastructures et une importante diversification de l'offre de produits touristiques, elle constitue dorénavant le pilier sûr d'une économie de services, qu'elle contribue dans le même temps à dynamiser.

L'évolution des modes de consommation de la clientèle dans le choix des hébergements ruraux, le niveau de fréquentation mais surtout les intérêts grandissants des populations agricoles pour un agritourisme financièrement plus rentable qu'une activité agricole dont les transformations au moment du changement ont profondément déstabilisé le secteur, ont suscité une conversion partielle des économies villageoises dans les activités d'accueil et de loisirs. La multiplication des chambres d'hôtes dans les villages et le développement des nouvelles formes d'agritourisme, liés en partie à une augmentation significative de la part d'une clientèle étrangère estimées à plus de $30 \%$ dont les facteurs de développement tiennent parfois plus à une situation agricole et sociale particulière qu'à des logiques économiques et environnementales plus traditionnelles, sont aujourd'hui, en grande partie responsables de la mise en place d'un tourisme dont le professionnalisme manque encore largement de sûreté. Peu ou pas formés, les acteurs agricoles, dans une grande majorité, se lancent dans un secteur touristique dont les modalités de gestion et d'organisation sont très différentes de celle du monde agricole.

La typologie que nous venons de présenter permet d'analyser les modalités de liaison entre les fonctions agricoles et touristiques. Celles-ci peuvent s'ordonner selon un net gradient est/ouest, des espaces aux dynamiques «indépendantes » les unes des autres (Silésie, Plateaux central de Moravie), aux espaces articulant les deux fonctions au sein d'usages très liés (Šumava, Sudètes). Cette typologie souligne l'existence de dynamiques contrastées dont les liens plus ou moins forts entre elles, tiennent avant toute chose, à une série de critères de nature différente. Certains sont d'environnemental et tiennent aussi bien à la qualité du potentiel agronomique qui, lorsqu'il est relativement faible, peut favoriser l'exercice d'une double activité agritouristique palliative, qu'à la présence d'une toute une série d'aménités potentiellement valorisées (paysages, cultures locales, etc.). L'inégale répartition de ces dotations factorielles, c'est-à-dire la nature, l'abondance et la qualité des aménités susceptibles d'être valorisées, par l'agriculture, le tourisme, ou par les deux, conduit naturellement à une différenciation de la dynamique des territoires, tous ne possédant pas les mêmes atouts. Ceci dit, hormis la présence de ces dotations, le développement du secteur touristique est aussi fortement corrélé aux capacités des espaces à les mettre en avant par la mise 
en place d'équipements et de services adéquats. Globalement, ce qui attire un récréant vers un espace est varié et combine souvent le naturel et l'artificiel, c'est-à-dire des potentialités mises en avant par une structuration des équipements. Mais tout ceci doit s'apprécier dans une analyse complexe où entrent en jeu de nombreux facteurs extra-récréatifs, qui donnent des impulsions ou des coups de freins (effets de modes par exemple pour l'agritourisme).

D'autres facteurs tiennent au positionnement de chaque territoire par rapport au continuum rural-urbain et à leur accessibilité. S'adossant à des structures d'exploitation « support » dont la valeur de la production est généralement négligeable par rapport aux bénéfices tirés de l'activité touristique (notamment dans l'ouest du pays), ils bénéficient d'une rente de situation favorable dans l'aire d'influence de certaines villes bavaroises ou autrichiennes. Globalement, les flux touristiques diminuent généralement avec l'éloignement de ces foyers émetteurs en raison de l’intervention conjointe des distances/temps, des distances/prix et des distances liées en partie aux infrastructures de transport.

D'autres facteurs tiennent à des logiques immobilières. Dans ce cas de figure, l'aménagement et la réhabilitation de bâtiments agricoles en gîtes s'inscrit, certes, dans une volonté de sauvegarde d'un patrimoine immobilier mais surtout, dans une optique de valorisation économique des bâtiments. Là aussi, l’inégale répartition de ce patrimoine, quelquefois épargné par les communistes dans les régions «agronomiquement » les moins rentables ou parfois détruit et transformé sur le modèle de la grande exploitation, conduit également à une différenciation importante de la dynamique touristique rurale tchèque, toutes les régions ne disposant pas d'un patrimoine architectural agraire digne d'être valorisé.

D'autres enfin, tiennent aux structures sociales des territoires ruraux et particulièrement à celles du monde agricole. La transition de 1989 et l'intégration à l'Union Européenne a profondément et durablement affecté la société agricole qui, dans un nouveau contexte économique libéral et compétitif, s'est heurtée aux problématiques de la rentabilité de son activité. Si dans les bassins de production les plus performants et les plus adaptés au modèle intensif, le soutien financier de Union Européenne a permis une modernisation assez rapide de la production agricole, ailleurs, dans les territoires les plus orientaux du pays, la question du surpeuplement agraire et de viabilité des exploitations, toujours pas réglés, est en partie occultée par le développement d'un tourisme «par défaut » dont le devenir est très souvent incertain.

\section{Bibliographie}

Busby (G.), Rendle (S.), 2000. - « The transition from tourism on farms to farm tourism », Tourism management, vol. 21, n 6, p. 635-642.

Chevalier (P.), 2008. - « Diversification et mutation des bases économiques dans l'espace rural tchèque », Revue d'Études Comparatives Est/Ouest, De la décollectivisation au développement local en Europe centrale et orientale, $\mathrm{n}^{\circ} 4$, vol. 39, p. 143-164.

Doucha (T.), Divila (E.), 2001 - « Farm Transformation and Restructuring in Czech Agriculture. After ten years ", in Ieda (O.) (éd.), The New Structure of the Rural Economy of Post-communist Countries, Slavik Research Center, Sapporo, p. 43-59.

Doucha (T.), 2004. - « Czech Agriculture and the EU accession. A Need for a New Strategy », Agricultural Economics, nº 50-3, p. 94-99.

Doucha (T.), Divila (E.), Fischer (M.), 2005. - « Land Use and Ownership and the Czech Farm Development », in Floriańczyk (Z.) et Czapiewski (K.) (éds), Rural Development Capacity in Carpathian Europe, vol. 3, Rural areas and development, Varsovie, p. 139-152.

Hudeč́ová (H.), Kučerová (E.), 2001. - « The Czech Republic in Rural Europe », Agricultural Economic, 47, 11, p. 481-489.

HudečKová (H.), LošTÁk (M.), 1993. - « Privatization in the Czech Agriculture », Eastern European Countryside, $\mathrm{n}^{\circ} 1$, p. 47-56. 
KučErová (E.), 2004. - « Rural Anticipation of the Welfare State in the Czech Republic », Eastern European Countryside, $\mathrm{n}^{\circ} 10$, p. 69-80.

Maurel (M.-C.), Lacquement (G.), 2007. - Agriculture et ruralité en Europe centrale, Paris, Éditions Aux lieux d'être, $190 \mathrm{p}$.

Majeroví (V.), 2000. - « Four Milestones in the Societal and Economic Development of Czech Agriculture », Czech Sociological Review, vol. 8 n², p. 157-176.

NeŠPor (Z.), 2006. - «The Son Has Ploughed”, But a Foreign Son. Five Case Studies on Transformation Strategies in Czech Agriculture after 1989 », Sociologický časopis = Czech Sociological Review, vol. 42 $n^{\circ}$ 6, p. 1171-1194.

Simon (A.), 2008. - «Agritourisme et recompositions des campagnes : une innovation inégalitaire », dans Ruralités nords-suds, inégalités, conflits, innovations, Paris, L'Harmattan, coll. "Itinéraires Géographiques ».

TěŠitel (J.), Kušová (D.), Bartoš (M.), 2003. - « Role of Tourism in Development of Rural Marginal Areas (Region of Šumava Mts in Czech Republic) », in Bański (J.) et Owsiński (J.) (éds.), Alternatives for European Rural Areas, Polska Akademia Nauk, IgiPZ, Varsovie, vol. 1, p. 81-92.

Zrinscak (G.), 2000. - «Y a-t-il un modèle agraire tchèque? », Enquêtes rurales, n 7, p. 75-96.

—, 1997. - Mutations des campagnes tchèques - Une décollectivisation pragmatique, Paris, Belin, coll. « Mappemonde », $256 \mathrm{p}$.

Cet article a été reçu le 23 mars 2009 et définitivement accepté le 24 juin 2009. 